\section{BRAZIULIAN JOURNAL}

OF MEDICAL AND BIOLOGICAL RESFARCH

www.bjournal.com.br
ISSN 0100-879X

Volume 43 (10) 914-1009 October 2010

BIOMEDICAL SCIENCES

AND

CLINICAL INVESTIGATION

Braz J Med Biol Res, October 2010, Volume 43(10) 964-968

doi: 10.1590/S0100-879X2010007500096

Functional changes of dendritic cells in hypersensitivity reactions to amoxicillin

C.M.F. Lima, J.T. Schroeder, C.E.S. Galvão, F.M. Castro, J. Kalil and N.F. Adkinson Jr.

The Brazilian Journal of Medical and Biological Research is partially financed by


Ministério da Educação



DFAPESP

Institutional Sponsors
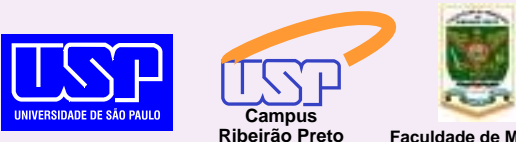

Ф SHIMADZU

GE Healthcare
Hotsite of proteomics metabolomics developped by:

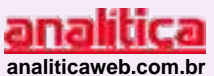

Thermo
SCIENTIFIC 


\title{
Functional changes of dendritic cells in hypersensitivity reactions to amoxicillin
}

\author{
C.M.F. Lima ${ }^{1,2}$, J.T. Schroeder ${ }^{1}$, C.E.S. Galvão², F.M. Castro², \\ J. Kalit ${ }^{2}$ and N.F. Adkinson Jr. ${ }^{1}$
}

${ }^{1}$ Division of Allergy and Immunology, Department of Medicine, Johns Hopkins Asthma and Allergy Center, Johns Hopkins University, Baltimore, MD, USA

2Departamento de Alergia e Imunologia Clínica, Hospital das Clínicas, Universidade de São Paulo, São Paulo, SP, Brasil

\begin{abstract}
A better understanding of dendritic cell (DC) involvement in responses to haptenic drugs is needed, because it represents a possible approach to the development of an in vitro test, which could identify patients prone to drug allergies. There are two main DC subsets: plasmacytoid DC ( $\mathrm{pDC}$ ) and myeloid DC (mDC). $\beta$-lactams form hapten-carrier conjugates and may provide a suitable model to study DC behavior in drug allergy reactions. It has been demonstrated that drugs interact differently with DC in drug allergic and non-allergic patients, but there are no studies regarding these subsets. Our aim was to assess the functional changes of $\mathrm{mDC}$ and $\mathrm{pDC}$ harvested from an amoxicillin-hypersensitive 32-year-old woman who experienced a severe maculopapular exanthema as reflected in interleukin-6 (IL-6) production after stimulation with this drug and penicillin. We also aim to demonstrate, for the first time, the feasibility of this method for dendritic cell isolation followed by in vitro stimulation for studies of drug allergy physiopathology. DC were harvested using a double Percoll density gradient, which generates a basophil-depleted cell (BDC) suspension. Further, pDC were isolated by blood DC antigen 4-positive magnetic selection and gravity filtration through magnetized columns. After stimulation with amoxicillin, penicillin and positive and negative controls, IL-6 production was measured by ELISA. A positive dose-response curve for IL-6 after stimulation with amoxicillin and penicillin was observed for $\mathrm{pDC}$, but not for $\mathrm{mDC}$ or BDC suspension. These preliminary results demonstrate the feasibility of this methodology to expand the knowledge of the effect of dendritic cell activation by drug allergens.
\end{abstract}

Key words: Dendritic cells; $\beta$-lactam hypersensitivity; Plasmacytoid dendritic cells; Drug allergy

\section{Introduction}

Adverse reactions to drugs are among the leading causes of iatrogenic morbidity and mortality. Severe T cell-mediated drug hypersensitivity reactions are a major problem both in drug development and in clinical practice. The current in vitro methods to confirm diagnosis and to indentify the culprit drug are still unreliable and have not been standardized. $\beta$-lactam antibiotics, the most common agents involved in allergic reactions to drugs, form haptencarrier conjugates with proteins that can be processed and presented by antigen-presenting cells (APC) to naive $T$ lymphocytes (1). In hypersensitive subjects, the immune system develops either Th2-type responses or delayed Th1-type responses and $\beta$-lactams provide the best model to analyze both types of reactions (2).

The involvement of T cells in these reactions has been studied (3) but little is known about the initial steps in the hypersensitivity response, consisting of drug allergen presentation to the lymphocytes, which is mediated by the innate immune response and served mainly by dendritic cells (DC). DC are professional APC, essential for initiating T-cell responses (4).

There are two main pathways of DC ontogeny from hematopoietic progenitor cells. One pathway generates plasmacytoid $\mathrm{DC}(\mathrm{pDC})$ while the other generates myeloid $\mathrm{DC}$ (mDC) (5). In human blood, there are two major groups based on surface antigens of immature DC (imDC), namely $\mathrm{CD}^{2} 3^{+}, \mathrm{CD}^{\circ} \mathrm{c}^{-}, \mathrm{CD} 303$ (blood DC antigen [BDCA]-2) ${ }^{+}$, CD304 (BDCA-4) ${ }^{+}$pDC and the CD123 low , CD11 high, CD1c $(\mathrm{BDCA}-1)^{+} \mathrm{mDC}(6)$.

DC can secrete a diversified panel of chemokines that attract different cell types at different times of the immune response (5). They also express a unique set of costimu-

Correspondence: C.M.F. Lima, Rua Padre Machado, 96, Apto. 52, 04127-000 São Paulo, SP, Brasil. Fax: +55-11-3069-2975.

E-mail: mafracynthia@hotmail.com

Received April 27, 2010. Accepted September 1, 2010. Available online September 24, 2010. Published October 18, 2010. 
latory molecules, which permit the activation of naive $T$ cells, launching the primary immune response and polarizing those cells into Th1, Th2, T-regulatory cells, Th9, or Th17 (7). Their abilities are regulated in a process known as maturation, by which they modulate the tolerant or the effector immune responses mediated by the different subtypes of T lymphocytes.

Furthermore, imDC and semi-mature DC (the so-called partially matured DC) have been related to tolerant immunologic responses, while mature $\mathrm{DC}$ have been related to effector responses, but this concept seems to be more complex, with the probable involvement of other unknown factors. Semi-mature DC have been characterized as those that do not produce proinflammatory cytokines despite the expression of $\mathrm{MHC}$ and co-stimulatory molecules (8).

Over the past years, some authors have investigated DC behavior in immune responses to drugs, based on the hypothesis that the involved mechanism lies in very early events that include the interactions between imDC and the drug itself (4).

Recent studies support the hypothesis that drugs interact differently with DC in drug-allergic and non-allergic patients and there is a body of evidence showing that the immunologic mechanism involved in the interactions between imDC and the drug could modify the maturative status of these imDC, which could affect the lymphocyte response to the drug. Sanderson et al. (9) have demonstrated for the first time that dendritic cells are capable of activating intracellular sulfamethoxazole and its metabolite nitroso sulfamethoxazole, which is more reactive in both chemical and immunological terms. These investigators argued that this is an immunologically important pathway that is highly relevant to hypersensitivity, and that the potential for crosstalk between drug metabolism and intracellular signaling in $\mathrm{DC}$ is an unexplored area that requires further investigation within the context of drug hypersensitivity.

Little is known about whether drugs or drug metabolites may act to generate signals that lead to activation of DC. Few previous studies regarding DC interaction with drugs have used peripheral blood mononuclear cells or DC generated from monocytes. Since none of them used purified DC subsets, the behavior of DC subsets regarding hypersensitivity responses to drugs remains unknown.

The exclusive expression of CD304 (BDCA-4) on pDC allows their direct isolation. Freshly isolated CD1c (BDCA$1)^{+}, \mathrm{CD} 141(\mathrm{BDCA}-3)^{+} \mathrm{mDC}$ and monocytes do not express CD304 (BDCA-4). Like CD304 (BDCA-4), CD303 (BDCA-2) is specifically expressed on pDC (10).

The aim of the present report is to demonstrate the interaction of amoxicillin and DC subsets isolated from the peripheral blood of a hypersensitive patient, by means of IL-6 production measurement after stimulation with this drug and penicillin. We also demonstrate for the first time the feasibility of a cell purification method using blood DC antigen 4-positive magnetic selection and gravity filtration through a magnetized column for studies regarding the physiopathology of drug allergy.

\section{Material and Methods}

\section{Patient selection}

A 32-year-old female patient who experienced a severe generalized maculopapular exanthema and facial angioedema in response to amoxicillin 10 days earlier was invited to participate in this study after giving informed written consent. The cutaneous lesions appeared on the 3rd day of amoxicillin treatment and she denied the use of any other medication during this period, as well as any previous history of adverse drug reactions.

The present assay was performed at the Johns Hopkins Asthma and Allergy Center, Johns Hopkins University, Baltimore, USA.

\section{Dendritic cell purification}

After venipuncture, a $150-\mathrm{mL}$ blood specimen was processed as described previously $(11,12)$. Briefly, this first involved the preparation of mixed leukocyte suspensions using a double Percoll (Bio-Sciences AB, Sweden; $1.075 / 1.081 \mathrm{~g} / \mathrm{mL}$ ) density centrifugation protocol (13). The basophil-depleted mononuclear cells (BDC) that floated on the lesser Percoll density were then recovered and washed free of platelets by performing 4 low-speed centrifugations (each $100 \mathrm{~g}, 10 \mathrm{~min}$ ) after each wash. pDC were then purified using blood DC antigen 4-positive selection (BDCA-4; Miltenyi Biotec, USA). Gravity filtration through magnetized columns attached to a MidiMACS magnet (Miltenyi Biotec) facilitated the purification of this cell type. For $\mathrm{pDC}$, this involved four washes to remove unbound (i.e., BDCA4 negative) cells and then removal of the column from the magnet and dislodging the pDC by adding buffer. An analysis of pDC suspensions prepared in this manner has previously indicated greater than $95 \%$ purity, as determined by BDCA-2 staining $(12,14)$.

Cells not retained in the column during BDCA-4 selection (i.e., the BDCA-4 flow through) were subsequently used to isolate $\mathrm{mDC}$ with BDCA-1 selection (Miltenyi Biotec). This first involved depleting cell suspensions of CD19+ $B$ cells and then a second pass through a different column to select for $\mathrm{BDCA}-1^{+} \mathrm{mDC}$. An analysis of $\mathrm{mDC}$ suspensions prepared in this manner had previously indicated greater than $95 \%$ purity, as determined by BDCA-1 staining (12).

Cells were counted using a Spiers-Levy chamber (Thomas Scientific, USA).

\section{Cell culture conditions}

All cultures were performed in conditioned-Iscove modified Dulbecco's medium (IMDM; Life Technologies Inc., USA) or C-IMDM (IMDM supplemented with 5\% heatinactivated fetal calf serum (FCS, $30 \mathrm{~min}$ at $56^{\circ} \mathrm{C}$; Sigma), $1 X$ non-essential amino acids, and $10 \mu \mathrm{g} / \mathrm{mL}$ gentamicin, 
pH 7.2-7.4 (Life Technologies Inc.).

BDC fractions (containing $T$ lymphocytes and monocytes), purified $\mathrm{pDC}$ and purified $\mathrm{mDC}$ suspensions were used for culture. The BDC fractions were cultured at approximately $5 \times 10^{6} / \mathrm{mL}$ in C-IMDM containing 5\% FCS. Approximately $1.25 \times 10^{6}$ BDCs were added to a 96-well plate $(0.125 \mathrm{~mL} /$ well; Corning Inc., USA) in a final volume of $0.25 \mathrm{~mL}$. The purified $\mathrm{pDC}$ and $\mathrm{mDC}$ were cultured at approximately 1 to $10^{5} / \mathrm{mL}$ in C-IMDM containing 5\% FCS. Approximately 25 to $50 \times 10^{3}$ purified $\mathrm{pDC}$ or $\mathrm{mDC}$ were added to a 96 -well plate $(0.125 \mathrm{~mL} /$ well $)$ in a final volume of $0.25 \mathrm{~mL}$. After incubating for $15 \mathrm{~min}$ to equilibrate to $37^{\circ} \mathrm{C}$ in the presence of $5 \% \mathrm{CO}_{2}$, an equal volume of stimulus or C-IMDM was added into duplicated cultures. Amoxicillin was used as the stimulus at pre-determined concentrations, i.e., 500,100 and $10 \mu \mathrm{g} / \mathrm{mL}$. To achieve these concentrations, a 500-mg amoxicillin capsule (GlaxoSmithKline, UK) was diluted in $50 \mathrm{~mL}$ saline and underwent a $\mathrm{pH}$ correction to approximately neutral, resulting in a uniform suspension of $12.5 \mathrm{mg} / \mathrm{mL}$ aliquots. This was subsequently diluted with C-IMDM.

Vancomycin hydrochloride (Novaplus, USA) was used as negative control at $5,50,250$, and $500 \mu \mathrm{g} / \mathrm{mL}$. Lyophilized vancomycin hydrochloride was diluted in $10 \mathrm{~mL}$ saline and used in $50 \mathrm{mg} / \mathrm{mL}$ aliquots, progressively diluted with CIMDM to achieve the concentrations cited above.

The monoclonal anti-FceRI antibody (eBioscience, USA) at $1: 167(75 \mathrm{ng} / \mathrm{mL})$ was used as a positive control in all culture wells. CpG ODN-2216 type A class (100 nM; Tri-Link, USA) was added as a positive control to culture wells containing BDC and pDC suspensions. Peptidoglycan (Invitrogen Life Technologies, USA) at $10 \mu \mathrm{g} / \mathrm{mL}$ was used as a positive control only in culture wells containing $\mathrm{mDC}$ suspensions. Previous studies from our laboratory have demonstrated that peptidoglycan is more efficient in stimulating $\mathrm{mDC}$ suspension cells than is $\mathrm{CpG}$, a finding likely due to the absence of Toll-like receptor 9 (TLR9) in $\mathrm{mDC}$.

Because amoxicillin is a synthetic penicillin, crystalline penicillin G (Roerig Division of Pfizer Inc., USA) was used as an additional stimulus reconstituted in $8.2 \mathrm{~mL}$ of a standard diluent, in aliquots of $500,000 \mathrm{IU} / \mathrm{mL}$ and diluted with C-IMDM at concentrations of 20, 200, and 1000 IU/ $\mathrm{mL}$. After a 20-h incubation period, the supernatants were harvested and frozen at $-70^{\circ} \mathrm{C}$ to be ultimately assayed for cytokines. For this preliminary study we assayed IL-6 levels using ELISA (eBioscience ELISA commercial kit; eBioscience).

\section{Results}

\section{BDC suspensions}

IL-6 production was observed in BDC suspensions after stimulation with the anti-FceRI and CpG-positive controls. Anti-FceRI was a more potent stimulus of IL-6 production than $\mathrm{CpG}$. On the other hand, IL-6 production was not observed after stimulation with amoxicillin and penicillin, or after stimulation with vancomycin used as a negative antibiotic control.

\section{pDC suspensions}

The dose-response curve for IL-6 production after stimulation with penicillin had a higher magnitude than the curve observed after amoxicillin stimulation. The positive anti-FceRI control demonstrated more potent stimulation for this cell suspension than CpG (Figure 1).

\section{mDC suspensions}

The purified mDC suspension only secreted IL-6 after stimulation with positive anti-FceRI controls and peptidoglycan. The latter was more potent than the former. No IL-6 secretion was observed after stimulation with vancomycin as the negative control or after stimulation with amoxicillin and penicillin (Figure 2).

\section{Discussion}

We propose the use of a standardized DC subset isolation method, comprised of magnetic selection and gravity filtration, in studies regarding drug hypersensitivity reaction mechanisms.

This method provides a pDC suspension with $95 \%$ homogeneity (12), which is high compared to other methods. This DC subset is very difficult to use because of its low frequency in peripheral blood. Also the potential for contamination with endotoxin from some biological substances used as a stimulus, like mite or milk proteins, has been a challenge for in vitro studies regarding DC immunobiology, since these substances may activate TLR leading to false-positive results. This contamination, however, does not seem to occur with drugs.

In this assay, we observed a positive dose-response curve for IL- 6 production by pDCs harvested from a hypersensitive subject after stimulation with penicillin and amoxicillin. However, it was not observed on mDC and BDC suspensions, despite the positive response after stimulation with positive controls.

In order to exclude toxic effects or non-specific stimuli induced by drugs in the DC subsets, vancomycin was used as negative control, since the subject did not have any history of vancomycin use.

The amoxicillin concentration used to stimulate DC in this assay was based on the studies of Rodriguez-Pena et al. (4), using DC cultivated from monocytes. These investigators suggested an active influence of amoxicillin on the maturational status of DC and demonstrated phenotypic and functional changes in DC cultivated from monocytes from hypersensitive patients, after in vitro stimulation with amoxicillin. In their study, amoxicillin seemed to direct DC from sensitive patients to a semimature phenotypic status, with increased expression of maturation and activation 


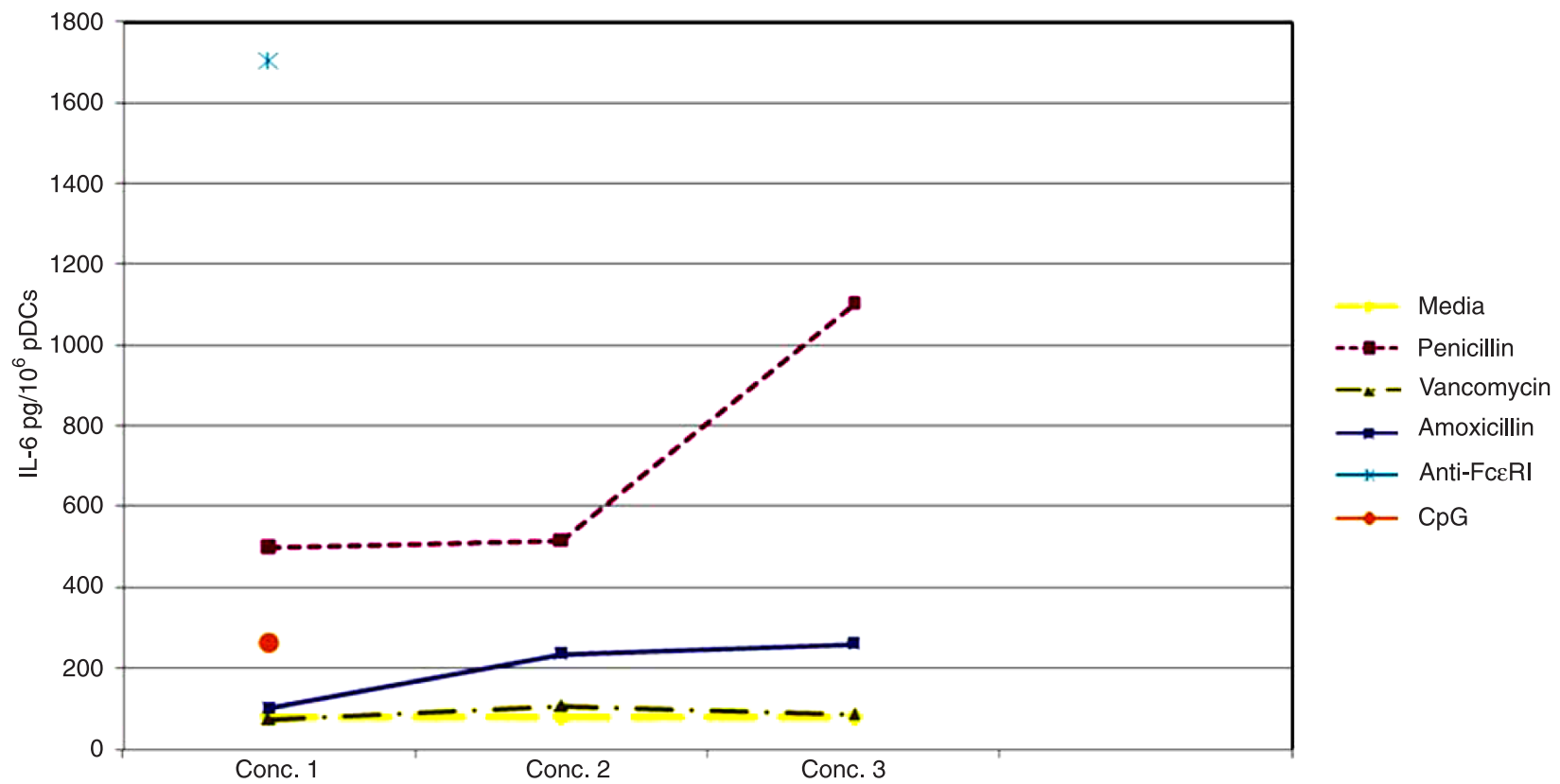

Figure 1. Concentration of IL-6 (pg/106 cells) produced by the suspension of plasmacytoid dendritic cells (pDC). Concentration (Conc.) 1: $20 \mathrm{IU} / \mathrm{mL}$ penicillin, $5 \mu \mathrm{g} / \mathrm{mL}$ vancomycin, $5 \mu \mathrm{g} / \mathrm{mL}$ amoxicillin, $75 \mathrm{ng} / \mathrm{mL}$ anti-FceRI/mL, and $100 \mathrm{nM}$ CpG. Concentration 2: $200 \mathrm{IU} /$ $\mathrm{mL}$ penicillin, $50 \mu \mathrm{g} / \mathrm{mL}$ vancomycin, and $50 \mu \mathrm{g} / \mathrm{mL}$ amoxicillin. Concentration 3: $1000 \mathrm{IU} / \mathrm{mL}$ penicillin, $250 \mu \mathrm{g} / \mathrm{mL}$ vancomycin, and $250 \mu \mathrm{g} / \mathrm{mL}$ amoxicillin.

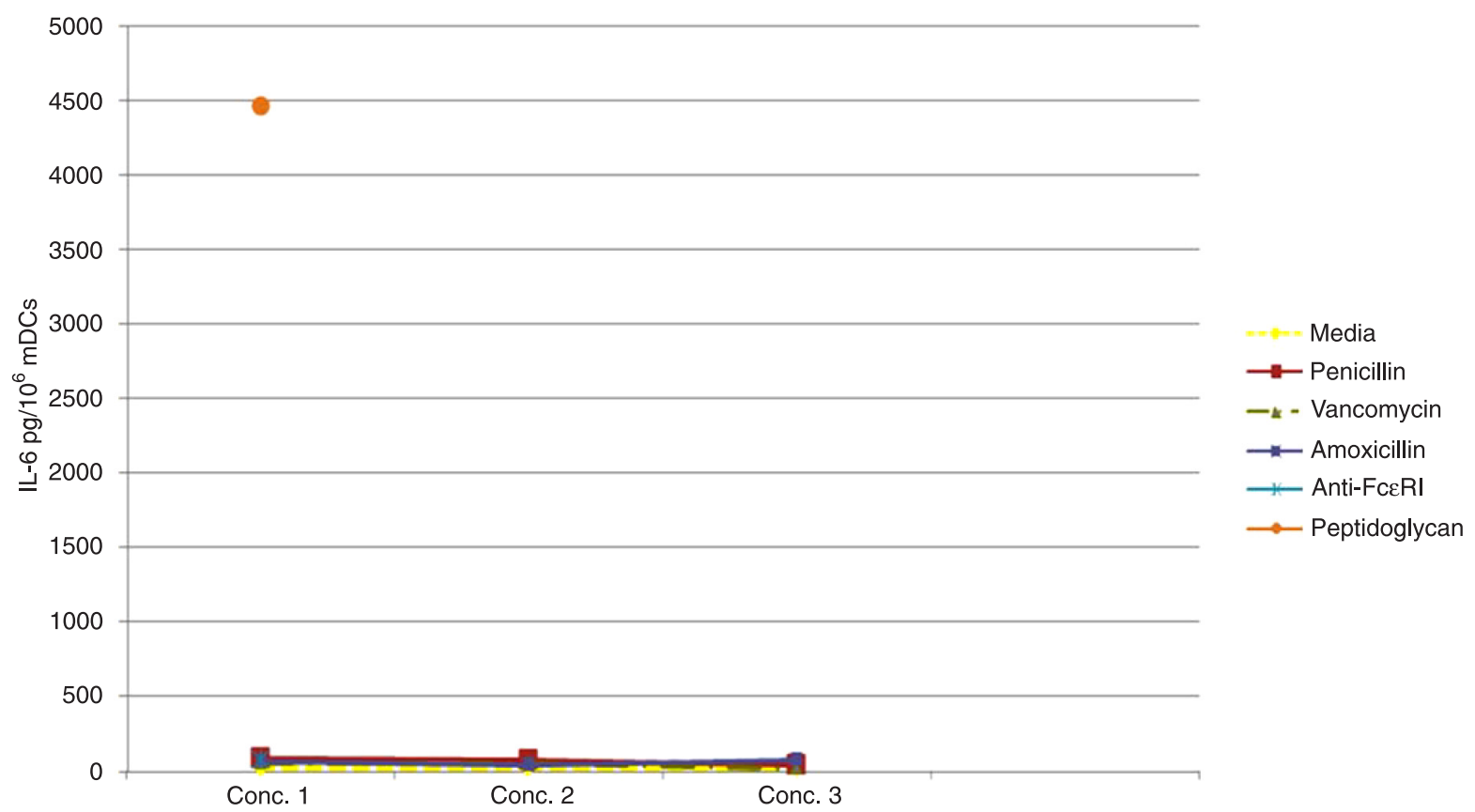

Figure 2. Concentration of IL-6 (pg/106 cells) produced by the suspension of myeloid dendritic cells (mDC). Concentration (Conc.) 1 : $20 \mathrm{IU} / \mathrm{mL}$ penicillin, $5 \mu \mathrm{g} / \mathrm{mL}$ vancomycin, $5 \mu \mathrm{g} / \mathrm{mL}$ amoxicillin, $75 \mathrm{ng} / \mathrm{mL}$ anti-FcERl/mL, and $10 \mu \mathrm{g} / \mathrm{mL}$ peptidoglycan. Concentration 2 : $200 \mathrm{IU} / \mathrm{mL}$ penicillin, $50 \mu \mathrm{g} / \mathrm{mL}$ vancomycin, and $50 \mu \mathrm{g} / \mathrm{mL}$ amoxicillin. Concentration 3: $1000 \mathrm{IU} / \mathrm{mL}$ penicillin, $250 \mu \mathrm{g} / \mathrm{mL}$ vancomycin, and $250 \mu \mathrm{g} / \mathrm{mL}$ amoxicillin. 
markers (HLA-DR, CD86, and CD80) and an increased endocytosis ability. However, amoxicillin was not capable of inducing complete maturation of these culture-derived DC when compared with other agents that interact directly with specific receptors such as lipopolysaccharide and TNF. This finding is consistent with the semi-mature status described by Lutz and Schuler (8) and leads us to speculate that amoxicillin might play a fundamental role in triggering the maturational process, or in the generation of signals in patient imDC that could induce the release of inflammatory mediators that activate DC (4). These investigators also suggest that, in an appropriate environment, amoxicillin acts not only as an antigen but also as a maturation stimulus.

In the present study, amoxicillin was not as potent a stimulus as the positive control or penicillin. Surprisingly, the latter stimulus was more potent than amoxicillin for pDC suspensions, and the IL-6 values were higher than the positive control CpG-ODN, even though the subject had denied any history of previous reaction to penicillin.

\section{References}

1. Weltzien HU, Moulon C, Martin S, Padovan E, Hartmann U, Kohler J. T cell immune responses to haptens. Structural models for allergic and autoimmune reactions. Toxicology 1996; 107: 141-151.

2. Schnyder B, Pichler WJ. Skin and laboratory tests in amoxicillin- and penicillin-induced morbilliform skin eruption. Clin Exp Allergy 2000; 30: 590-595.

3. Nassif A, Bensussan A, Boumsell L, Deniaud A, Moslehi H, Wolkenstein $P$, et al. Toxic epidermal necrolysis: effector cells are drug-specific cytotoxic T cells. J Allergy Clin Immunol 2004; 114: 1209-1215.

4. Rodriguez-Pena R, Lopez S, Mayorga C, Antunez C, Fernandez TD, Torres MJ, et al. Potential involvement of dendritic cells in delayed-type hypersensitivity reactions to beta-lactams. J Allergy Clin Immunol 2006; 118: 949-956.

5. Piqueras B, Connolly J, Freitas H, Palucka AK, Banchereau J. Upon viral exposure, myeloid and plasmacytoid dendritic cells produce 3 waves of distinct chemokines to recruit immune effectors. Blood 2006; 107: 2613-2618.

6. Liu YJ. IPC: professional type 1 interferon-producing cells and plasmacytoid dendritic cell precursors. Annu Rev Immunol 2005; 23: 275-306.

7. Akdis CA, Akdis M. Mechanisms and treatment of allergic disease in the big picture of regulatory T cells. J Allergy Clin Immunol 2009; 123: 735-746.

8. Lutz MB, Schuler G. Immature, semi-mature and fully mature dendritic cells: which signals induce tolerance or immunity? Trends Immunol 2002; 23: 445-449.

9. Sanderson JP, Naisbitt DJ, Farrell J, Ashby CA, Tucker MJ, Rieder MJ, et al. Sulfamethoxazole and its metabolite nitroso
However, further studies will be necessary to optimize the penicillin and amoxicillin concentration to be used for stimulation. To our knowledge, these antibiotics have never been used for in vitro DC stimulation.

These results encouraged us to develop a similar study with a greater number of subjects in order to study pDCs behavior on drug hypersensitive reactions. The interaction of DC with drugs is an emerging area of research, which can bring new insights, not only for the understanding of adverse drug reaction physiopathology, but also to improve the current knowledge of DC immunobiology.

Additionally, Aubert et al. (15) demonstrated that DC pulsed with neuromuscular blocker agents were able to induce a significant proliferative T-cell response in two hypersensitive subjects. This suggests that a better understanding of the DC activation mechanisms in drug hypersensitivity reactions may lead to improved efficacy of the lymphocyte transformation test in the identification of the culprit drug. sulfamethoxazole stimulate dendritic cell costimulatory signaling. J Immunol 2007; 178: 5533-5542.

10. Dzionek A, Fuchs A, Schmidt P, Cremer S, Zysk M, Miltenyi S, et al. BDCA-2, BDCA-3, and BDCA-4: three markers for distinct subsets of dendritic cells in human peripheral blood. J Immunol 2000; 165: 6037-6046.

11. Schroeder JT, MacGlashan DW Jr, Kagey-Sobotka A, White JM, Lichtenstein LM. IgE-dependent IL-4 secretion by human basophils. The relationship between cytokine production and histamine release in mixed leukocyte cultures. $J$ Immunol 1994; 153: 1808-1817.

12. Le T, Tversky J, Chichester KL, Bieneman AP, Huang SK, Wood RA, et al. Interferons modulate Fc epsilon RI-dependent production of autoregulatory IL-10 by circulating human monocytoid dendritic cells. J Allergy Clin Immunol 2009; 123: 217-223.

13. Chen $\mathrm{YH}$, Bieneman AP, Creticos PS, Chichester KL, Schroeder JT. IFN-alpha inhibits IL-3 priming of human basophil cytokine secretion but not leukotriene $\mathrm{C} 4$ and histamine release. J Allergy Clin Immunol 2003; 112: 944-950.

14. Schroeder JT, Bieneman AP, Xiao H, Chichester KL, Vasagar K, Saini S, et al. TLR9- and FcERI-mediated responses oppose one another in plasmacytoid dendritic cell by downregulating receptor expression. J Immunol 2005; 175: 57245731.

15. Aubert N, Mertes PM, Janaszak M, Moneret-Vautrin DA, Laxenaire MC, Kanny G, et al. Dendritic cells present neuromuscular blocking agent-related epitopes to $T$ cells from allergic patients. Allergy 2004; 59: 1022-1023. 